\title{
Adolescent Primary Care Visit Patterns
}

\author{
James D. Nordin, $M D, M P H^{1}$ \\ Leif I. Solberg, $M D^{1}$ \\ Emily D. Parker, $M P H, P b D^{1}$ \\ 'HealthPartners Research Foundation, \\ Minneapolis, Minnesota
}

Conflicts of interest: none reported

\section{CORRESPONDING AUTHOR}

James D. Nordin, MD, MPH

HealthPartners Research Foundation

PO Box 1524 MS \#21111R

Minneapolis, MN 55440-1524

james.d.nordin@healthpartners.com

\begin{abstract}
PURPOSE Many clinical preventive care services are recommended for adolescents. Little is known about whether most adolescents have a sufficient number of preventive care services visits over time to receive those services. We wanted to measure how frequently adolescents who are insured either through private insurance or government programs have preventive vs nonpreventive care visits.
\end{abstract}

METHODS We conducted a retrospective descriptive analysis based on claims data from a large health plan in Minnesota with about 700,000 members. All study patients were aged 11 to 18 years between January 1, 1998, and December 31, 2007. Our outcome measure was rates of preventive and nonpreventive care visits.

RESULTS One-third of adolescents with 4 or more years of continuous enrollment had no preventive care visits from age 13 through 17 years, and another 40\% had only a single such visit. Nonpreventive care visits were more frequent in all age-groups, averaging about 1 per year at age 11 years, climbing to about 1.5 per year at age 17 years. Differences in rates between government insurance and commercial insurance were small. In older adolescence, girls had more preventive care visits and more nonpreventive care visits than did boys.

CONCLUSIONS Most adolescents come in infrequently for preventive care visits but more often for nonpreventive care visits. We recommend using the same approach in adolescence for preventive care that is being used in adults: the nomissed-opportunities paradigm. All visits by adolescents should be viewed as an opportunity to provide preventive care services, and systems should be set up to make that possible, even in busy practices with short encounters with a clinician.

Ann Fam Med 2010;8:511-516. doi:10.1370/afm.1188.

\section{INTRODUCTION}

$\mathrm{D}$ uring the past 20 years, the number of clinical preventive care services recommended for adolescents by national organizations have increased considerably. The American Academy of Pediatrics' Bright Futures ${ }^{1}$ and the American Medical Association (AMA) Guidelines for Adolescent Preventive Services ${ }^{2}$ both recommend visits for annual preventive care services for all patients aged 11 to 21 years. Little is known, however, as to how often adolescents actually visit a medical office or their reasons for those visits. Thus, there is little agreement about how best to deliver these preventive care services during those visits and scant information on whether most adolescents have enough preventive care medical encounters during the course of their teenage years to receive the services recommended. It is also unknown whether they have a sufficient number total encounters (preventive and otherwise) to receive all of the recommended services if the services are offered at every visit. Disparities in care based on socioeconomic status have been found in many areas of medicine and may exist here.

Some data exist suggesting that 13-to 18-year-olds visit the doctor less often than most age-groups. In a cross-sectional study, Ma et al reviewed National Ambulatory Medical Care Survey (NAMCS) medical visit data for the period from 1993-2000. ${ }^{3}$ They found that adolescents aged 13 
to 18 years averaged 1.9 visits a year, lower than any other age-group except adolescents aged 19 to 24 years. The National Health Interview Survey (NHIS) found that in 1995, 90\% of insured adolescents and $75 \%$ of uninsured adolescents had at least 1 physician contact in the past year. ${ }^{4}$ No longitudinal data exist for adolescents over a several year period.

This study was designed to quantify type and frequency of adolescent visits. Minnesota requires documentation of an up-to-date status of immunizations at entrance to grade 7 (age 12 years for most children), and a physical examination is strongly encouraged. We were interested in the effect of state requirements on preventive care visits, and even more interested in the visit rates after that landmark year. We were also interested in the impact of the several covariates we could test: age, sex, and insurance type (as a dichotomous variable for socioeconomic status).

Specifically, this study was designed to answer the following questions:

1. What is the frequency of preventive care and other medical visits by adolescents for each year from their 11th to their 18th birthday (cross-sectional study)?

2. What is the frequency of preventive care and other medical visits over the entire period from age 13 to 18 years for those with continuous enrollment in a single insurance plan for 4 or more years (longitudinal study)?

3. Do these patterns vary according to the covariates of insurance type (public compared with private pay), sex, or age?

We tested the following hypotheses: (1) adolescents have few preventive care visits, (2) adolescents have few nonpreventive care visits, (3) adolescents with continuous coverage will have higher visit rates of both visit types than those with shorter coverage, (4) girls will have more visits than boys, and (5) socioeconomic status will matter, that is, patients insured through government-based programs will have a rate of both visit types that is lower than the rate for those with commercial insurance

\section{METHODS}

\section{Data Sources}

We performed an analysis of administrative data from HealthPartners (a Minnesota health plan with 750,000 members), assessing primary care visit rates for all adolescent health plan members who had coverage from January 1, 1998, through December 31, 2007. This analysis evaluated rates for preventive care visits and for all other visits that generated a claim with in- and out-of-network clinicians in a primary care department (pediatrics, family medicine, internal medicine, or gynecology). For many years, the health plan has covered annual well-child visits. Current Procedural Terminology diagnosis (CPT/DX) codes 99381-99397 were used to define preventive care visits. Other visits were defined as all clinician visits that did not have one of these preventive care visit CPT/DX codes. All data were extracted from the HealthPartners claims database, and no chart review was performed. Insurance type was determined by examining claims data.

This study was reviewed, approved, and monitored by the HealthPartners Institutional Review Board.

\section{Study Sample}

The potentially eligible population included all HealthPartners members who had at least one 11-year through 18 -year birthday during the period being studied. HealthPartners enrolls about $15 \%$ of Minnesotans. There were 300,866 adolescent plan members between 1998 and 2007 who were considered for these analyses. Of the HealthPartners adolescent population, 93\% was covered by commercial insurance and $7 \%$ by government insurance programs. For the state of Minnesota, the figures for the same period are $72 \%$ and $21 \%$, respectively, with $7 \%$ having no insurance. Because only those with insurance are found in this data set, these data represent a best-case scenario.

To be included in the 1 -year cross-sectional analyses by age, members had to be enrolled in the health plan for at least 1 full year, allowing only an administrative gap of up to 32 days between birthdays. In each calendar year about one-half the adolescents met that criterion. Those with fewer than 333 days of continuous enrollment were not included in the study.

For longitudinal analyses looking at patterns in preventive care visits over time, members had to be enrolled in the health plan for 4 or more years with no more than one 32-day gap per year between their 13 th and 18 th birthday. This requirement eliminated $86.7 \%$ of adolescent members. Insurance type was used as a dichotomous variable to assess the impact of socioeconomic status on visit frequency.

\section{Statistical Methods}

All analyses were done using SAS 9.1.3 (SAS Institute, Cary, North Carolina). For the cross-sectional study sample we computed frequencies, means, and distributions stratified by ages 11 through 18 years. We used linear regression to test for trends with age.

For the longitudinal study we used a 2 -sided $t$ test to compare the average number of visits at each year of age between insurance types (commercial and government/Medicaid), as well as between male and female adolescents. We estimated adjusted visit rates for sex and insurance type using linear regression. 


\section{RESULTS}

We compared mean number of visits between the 2 study samples. The average number of nonpreventive care visits for adolescents aged 15, 16, and 17 years was higher in the cohort with 4 or more years of continuous insurance coverage. For example, among those aged 16 years in the cross-sectional study sample, the mean number of nonpreventive care visits was 1.36 and 1.47 for adolescents who had commercial and government insurance, respectively, and 1.73 and 1.68, respectively, among adolescents aged 16 years in the longitudinal cohort. These differences were statistically significant $(P<.001)$. Among the populations that had commercial and government insurance, there were $27 \%$ more and $14 \%$ more nonpreventive care visits, respectively in the 4 -year cohort. The continuous enrollment sample is not representative, but rather represents the absolute best possible conditions. Thus these data will represent the most complete care being given to adolescents.

\section{Cross-Sectional Analyses}

There were an average of 77,564 observations for each year of age in the cross-sectional analysis. Forty-nine percent of the adolescents observed were female.

The average number of preventive care visits per year for adolescents with either commercial or government insurance was highest in those aged 12 years $(0.42,0.45$ respectively, difference, $P=.814)$. For all other ages the mean number of preventive care visits per year ranged from 0.15 to 0.26 for commercially insured adolescents and 0.20 to 0.28 for governmentinsured adolescents. The difference in rates between these insurance types was small. In terms of age, only the differences between those aged 12 years and other ages are large.

Nonpreventive care visits were more frequent than preventive care visits in all age-groups, averaging about 1 per year at age 11 years, climbing to about 1.5 per year at age 17 years (trend for age, $P \leq .001$ commercial insured and $P \leq .001$ government insured). For those younger than 15 years, there were more nonpreventive care visits in government plan patients. At age 15 years and older, nonpreventive care visits were more common among those with commercial insurance (Figure 1).

\section{Longitudinal Analyses}

In the longitudinal analyses, we examined patterns in preventive care and nonpreventive care visits among the 40,043 adolescents who were continuously insured for at least 4 years by either commercial or government programs. Again, this group would be expected to get the most care.

The proportions of adolescents who had $0,1,2$, 3,4 , and 5 or more preventive care visits during the observation period are shown in Table 1 . Thirty percent of adolescents with either insurance type had no preventive care visits during this 4 - to 5 -year interval. Another $41 \%$ of commercially insured and $36 \%$ of government-insured adolescents had only 1 preventive care visit during this time. Only $1 \%$ of adolescents with commercial insurance and $2 \%$ of those with gov-

\section{Figure 1. Mean number of nonpreventive care visits by age and insurance type (commercial or govern-} ment) in a sample of adolescents between the ages of 11 and 18 years, HealthPartners, 1998-2007.

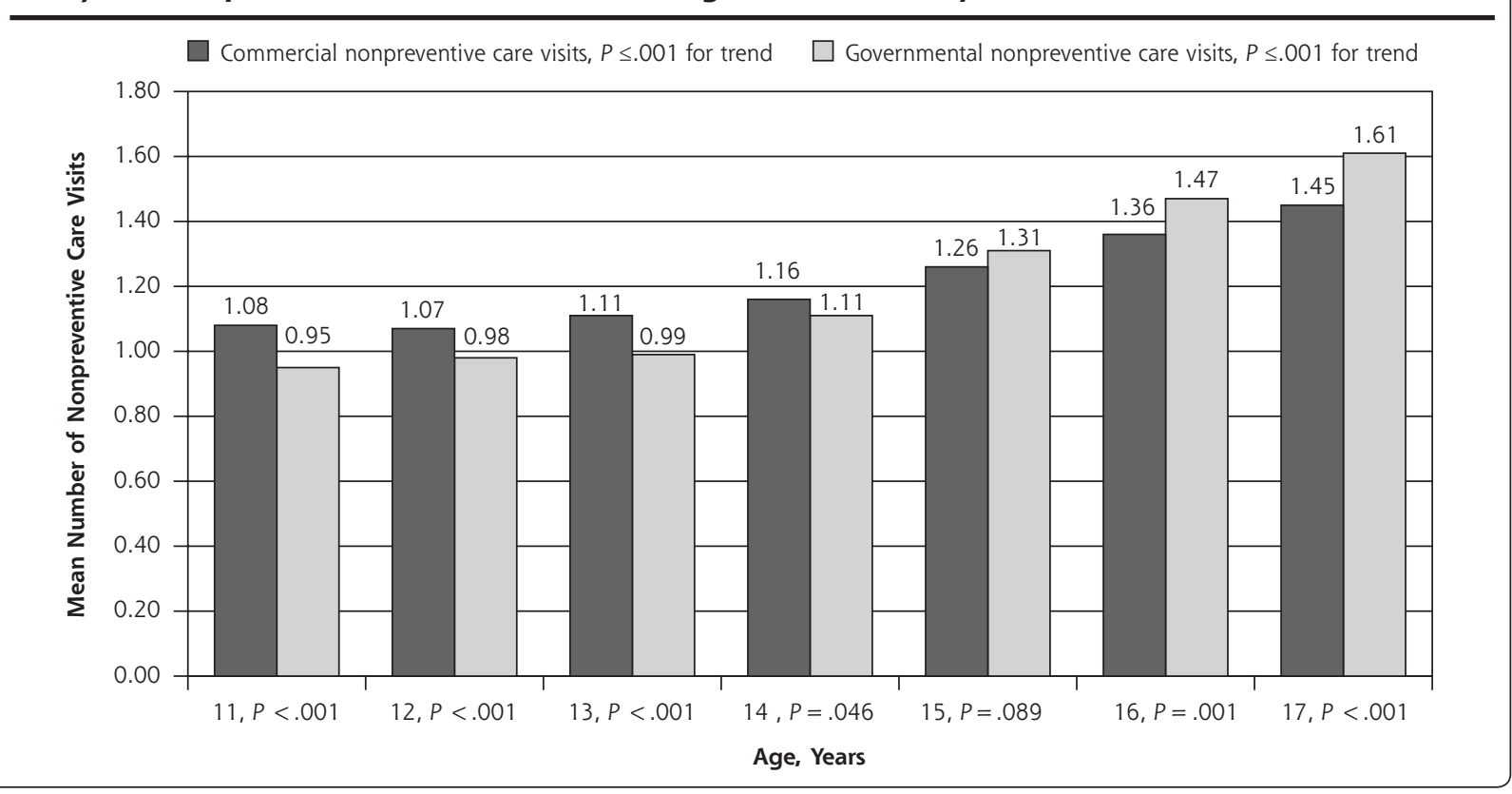




\section{Table 1. Patterns in Health Care Utilization and Insurance Among Adolescents Aged 13 to 18 With Continuous Health Insurance Enrollment, HealthPartners, 1997-2008}

\begin{tabular}{|c|c|c|c|c|c|c|}
\hline \multirow[b]{2}{*}{ Visit Characteristics } & \multicolumn{3}{|c|}{ Nonpreventive Care } & \multicolumn{3}{|c|}{ Preventive Care, \% } \\
\hline & Commercial, \% & Government, \% & & Commercial, \% & Government, $\%$ & \\
\hline \multicolumn{7}{|l|}{ Number of visits } \\
\hline 0 & 8 & 15 & & 30 & 30 & \\
\hline 1 & 9 & 13 & & 41 & 36 & \\
\hline 2 & 10 & 10 & & 22 & 21 & \\
\hline 3 & 9 & 9 & & 5 & 9 & \\
\hline 4 & 9 & 8 & & 1 & 2 & \\
\hline 5 to 10 & 34 & 25 & & 0 & 0 & \\
\hline \multirow[t]{2}{*}{$>10$} & 20 & 20 & & 0 & 0 & \\
\hline & No. & No. & $P$ Value $^{\mathrm{a}}$ & No. & No. & $P$ Value \\
\hline Mean total visits, No. & 6.83 & 6.41 & .028 & 1.07 & 1.18 & $<.001$ \\
\hline Mean visit rate per year & 1.50 & 1.47 & .570 & 0.23 & 0.27 & $<.001$ \\
\hline
\end{tabular}

ernment insurance had 1 preventive care visit each year.

These patterns differed by sex. The mean number of preventive care visits during the observation period was 1.16 for female adolescents and 0.99 for male adolescents $(P<.001)$. The mean preventive care visit rates per year during this period for female and male adolescents were 0.26 and $0.22(P<.001)$, respectively. The mean number of nonpreventive care visits was 7.73 for girls and 5.92 for boys $(P<.001)$. Finally, Table 2 shows the mean and standard deviation for preventive care and nonpreventive care visits, adjusted for sex and insurance type. This adjustment did not change the means significantly. Female adolescents averaged $16 \%$ more preventive care visits and 30\% more nonpreventive care visits than male adolescents

\section{DISCUSSION}

For members of a large Midwest health plan, we have documented that one-third of adolescents with 4 or more years of continuous enrollment had no preventive care visits from age 13 through 17 years, and another $40 \%$ had only 1 such visit. They all had insurance without a deductible or co-pay for such visits, and as long-term health plan members, there should have been minimal problems getting established with a source of care. It is also worth noting that there was no difference in preventive care visits between those on Medicaid and those with commercial insurance. This lack of difference is likely due to efforts by the health plan to minimize disparities, which include financial incentives for both clinicians and patients. As a part of the quality incentive, clinicians are paid a bonus if they reach specified goals for preventive care visits for government-insured patients, and the patients receive a Target gift certificate for completing a preventive care visit. In Minnesota, where immunization status has to be updated for school attendance at grade 7 and a physical is strongly encouraged, more than onehalf had a preventive care visit at age 11 or 12 years before entering the later period of preventive care visit drought. In contrast, most patients are seen for nonpreventive care visits, averaging 1 to 2 such visits per year.

Although some have studied this issue, none have reported on visit rates for the same individuals over time. All previous studies have been cross-sectional. Ma et al reviewed NAMCS medical visit data from 1993-2000. ${ }^{3}$ They found that adolescents aged 13 to 
18 years averaged 1.9 visits per year, lower than any other age-group except those aged 19 to 24 years (eg, those aged 25 to 64 years averaged 3.1, and those aged 65 years and older averaged 6.1). General medical examinations accounted for $7.5 \%$ of adolescent visits, but male adolescents had another $3.7 \%$ for physical examinations for extracurricular activities. These data are similar to what we found. Rand et al reviewed the 1994-2003 NAMCS data and found 9\% of adolescent visits were for preventive care, but the rate declined after age 14 years for female adolescents and after age 13 years for male adolescents. ${ }^{5}$ They found that "more than 3 times as many preventive care visits were made by early adolescents than by late adolescents." Again our results confirm this finding. In a prepaid health maintenance organization (Kaiser Portland) where insurance is not a limiting factor, $69 \%$ of adolescents aged 14 to 17 years had a primary care visit during 1 year, and $83 \%$ had a primary care visit during 2 years ( $85 \%$ for girls and $81 \%$ for boys). ${ }^{6}$ Primary care visits accounted for $90 \%$ of all visits. Those who made primary care visits averaged 2.5 such visits per year, a finding similar to ours.

Another national survey, the National Health Interview Survey (NHIS), found that in 1995, 90\% of insured adolescents had at least 1 physician contact in the past year (mean $=2.8$ ). This rate is slightly higher than what we found. ${ }^{4}$ Klein et al surveyed 6,748 adolescents in $1997 ; 85 \%$ said they had had a well-patient visit in the past year, $9 \%$ had a well-patient visit 1 to 2 years ago, and $6 \%$ had a well-patient visit more than 2 years ago. ${ }^{7}$ For those with no insurance, however, the rates were $76 \%, 12 \%$, and $12 \%$, respectively. This rate is a much higher than what we found and than found in previously cited papers. We believe our claims data are more accurate than patient survey data.

The problem with each of the above studies is that they report group averages only for short periods, which minimizes the serious differences among subgroups of adolescents, differences highlighted by the current study.

Improvement efforts will require different interventions in various subgroups and at every opportunity. In the current paradigm, most adolescent preventive care services are provided only at preventive care visits. All visits by adolescents should be viewed as an opportunity to provide preventive care services, and systems should be set up to make that possible, even in busy practices with short clinician encounters. Previsit planning can be done each day to determine what services are needed for each of the scheduled patients. Electronic medical records systems can be used to remind patients services are needed and recontact them if they are overdue, and to remind clinicians of preventive care services needed for each patient they see each day. Standing orders can be used so nurses can provide preventive care services even if the clinician neglects to order them.

This study has some weaknesses. Most important was the requirement for 4 or more years of continuous coverage for inclusion in the longitudinal analyses, which necessitated the need to collect continuous data from our database. (When a patient changes insurance programs, there is no way to know whether that patient has had a visit.) As a result, we undoubtedly selected a more stable, more compliant subset of the whole population, biasing our rates upward; thus, the samples included in these analyses represent an optimal situation. As poor as they are, these rates probably overestimate how well we are doing at achieving preventive care visits for the entire adolescent population. Another limitation is that a small amount of misclassification of visit types may have occurred. Even so, all of these data are likely to be more accurate than data collected from patient surveys requiring patient recall. Many will argue that conducting this study in an insured population is a limitation. Minnesota has a very high rate of insurance coverage. In addition, based on previous, unpublished work at HealthPartners Research Foundation, comparisons of the health plan membership with the composition of the metropolitan area suggest that health plan demographics mirror those of the metropolitan area. ${ }^{8,9}$ Finally, these data are from a single health plan in one metropolitan area and may not be generalizable to other areas and other health plans.

The AMA's Guidelines for Adolescent Preventive Services $^{2}$ and the American Academy of Pediatrics' Bright Futures ${ }^{1}$ both recommend annual preventive care services visits for all patients aged 11 to 21 years. These recommendations are being met by less than $2 \%$ of our sample. That adolescents are visiting the doctor for other reasons, however, argues strongly for using the same approach in adolescence increasingly being used in adults for preventive care, the no-missedopportunities paradigm.

To read or post commentaries in response to this article, see it online at http://www.annfammed.org/cgi/content/full/8/6/511.

Key words: Adolescent; delivery of health care; primary health care/ utilization; preventive health services

Submitted March 30, 2010; submitted, revised, July 1, 2010; accepted July 28, 2010.

Author contributions: The authors retained full control over the content, had full access to all the data in the study, and take responsibility for the integrity of the data and the accuracy of the data analysis. Each of the 3 authors participated in the design and conduct of the study; collection, management, analysis, and interpretation of the data; and preparation, review, or approval of the manuscript. 
Funding support: This study was funded by a grant from Partnership for Prevention.

Role of sponsor: The sponsor was involved in forming the concept of this study and reviewed the manuscript before it was submitted.

Acknowledgment: Ann Hanson produced the analytic data set from the HealthPartners claims data.

\section{References}

1. Recommendations for Preventive Pediatric Health Care. Elk Grove Village, IL: American Academy of Pediatrics. http://brightfutures .aap.org/pdfs/Guidelines_PDF/20-Appendices_PeriodicitySchedule .pdf. Accessed Jan 12, 2009.

2. AMA Guidelines for Adolescent Preventive Services. Chicago: American Medical Association. http://www.ama-assn.org/ama/upload /mm/39/gapsmono.pdf. Accessed Jan 12, 2009.

3. Ma J, Wang Y, Stafford RSUS. U.S. adolescents receive suboptimal preventive counseling during ambulatory care. J Adolesc Health. 2005;36(5):441.
4. Newacheck PW, Brindis CD, Cart CU, Marchi K, Irwin CE. Adolescent health insurance coverage: recent changes and access to care. Pediatrics. 1999;104(2 Pt 1):195-202

5. Rand CM, Shone LP, Albertin C, Auinger P, Klein JD, Szilagyi PG. National health care visit patterns of adolescents: implications for delivery of new adolescent vaccines. Arch Pediatr Adolesc Med. 2007;161(3):252-259.

6. O'Connor EA, Hollis JF, Polen MR, Lichtenstein E. Adolescent health care visits: opportunities for brief prevention messages. Eff Clin Pract. 1999;2(6):272-276.

7. Klein JD, Wilson KM, McNulty M, Kapphahn C, Collins KS. Access to medical care for adolescents: results from the 1997 Commonwealth Fund Survey of the Health of Adolescent Girls. J Adolesc Health. 1999:25(2):120-130.

8. Jonk YC, Call KT, Cutting AH, O'Connor H, Bansiya V, Harrison K. Health care coverage and access to care: the status of Minnesota's veterans. Med Care. 2005;43(8):769-774

9. Call KT, Lurie N, Jonk Y, Feldman R, Finch MD. Who is still uninsured in Minnesota? Lessons from state reform efforts. JAMA. 1997;278(14):1191-1195. 